\title{
Concentration Profiles of Colloidal Fluids in One-Dimensional Confinement
}

\author{
Oliver Bunk ${ }^{\mathrm{a}}$, Dillip K. Satapathy ${ }^{\mathrm{a}}$, Franz Pfeiffer ${ }^{\mathrm{ab}}$, Ana Diaz ${ }^{\mathrm{ac}}$, Christian David ${ }^{\mathrm{a}}$, Kim Nygårda, \\ Edith Perret ${ }^{a}$, Celestino Padestea ${ }^{a}$, Philip R. Willmott ${ }^{\mathrm{a}}$, Bruce D. Patterson ${ }^{\mathrm{a}}$, \\ Bernd Schmitt ${ }^{\mathrm{a}}$, and J. Friso van der Veen ${ }^{\star a d}$
}

\begin{abstract}
An X-ray diffraction method has been developed to derive concentration profiles of fluids under onedimensional confinement. A microfluidic array of channels is used as a container for the fluid under investigation. Thereby the ensemble average of many channels is studied rather than individual realizations of the system. Additionally, the X-ray scattering signal is enhanced and the radiation dose per fluid volume reduced. As an example, data are shown for a colloidal solution of silica particles of about $109 \mathrm{~nm}$ diameter under confinement in channels ranging from 280 to $615 \mathrm{~nm}$ width. The density profiles across the channels have been determined in a model independent way with a resolution in the $10 \mathrm{~nm}$ regime. When the electrostatic potential of the colloidal particles is shielded, we observe an oscillatory ordering-disordering behaviour of the colloidal particles as a function of the channel width, while the colloidal solution remains in the liquid state. This phenomenon has been suggested by surface force studies of hard-sphere fluids and also theoretically predicted, but has so far never been seen by direct measurements of the structure for comparable systems. The size of the fluid's constituents and of the confining cavities can be scaled down to below $100 \mathrm{~nm}$, opening up the possibility for the investigation of confinement effects in e.g. protein crystallization.
\end{abstract}

Keywords: Colloidal solution $\cdot$ Confinement $\cdot$ Microfluidics $\cdot$ Small angle X-ray scattering $\cdot$ X-ray diffraction

\section{Introduction}

Confined fluids are abundant in both nature and modern technology. Examples are water or oil in porous rock, wet chemistry production of nanostructures, tribology, especially lubrication, transport of pharmaceutical carriers through blood vessels and pores in biological membrane systems. Geometrical confinement can dramatically alter bulk

${ }^{*}$ Correspondence: Prof. J. F. van der Veen ${ }^{\text {ad }}$ E-mail: friso.vanderveen@psi.ch

aResearch Department Synchrotron Radiation and

Nanotechnology

Paul Scherrer Institut

$\mathrm{CH}-5232$ Villigen PS

${ }^{b}$ Ecole Polytechnique Fédérale de Lausanne

$\mathrm{CH}-1015$ Lausanne

cPresent address: European Synchrotron Radiation

Facility

B.P. 220

38043 Grenoble Cedex

France

dETH Zürich

$\mathrm{CH}-8093$ Zürich properties of fluids. Best known examples are a dramatic increase in viscosity and reduced diffusion. In a narrow confined space, the fluid can change its phase behaviour and may become even solid-like. ${ }^{[1,2]}$ It is well known that the presence of a single wall induces layering of the fluid. ${ }^{[3-6]}$ The presence of two opposing walls is expected to strongly favour layering whenever their distance happens to be an integer number of fluid layers and to disfavour layering when this is not the case. Evidence for these effects has been obtained through the measurement of oscillatory structural forces in the surface force apparatus (SFA). ${ }^{[7,8]}$ With a few exceptions, ${ }^{\left[{ }^{[-13]}\right.}$ a direct measurement of the fluid's density profile between the walls has proven to be difficult for a number of reasons:

i) it is hard to gain access to the narrow confined space with an experimental probe,

ii) the sample volume is extremely small, in the range of femtoliters, and

iii) the shape of the fluid's container may be too complex and not precisely known.

Determining the density profile of a fluid across the confining container with high resolution has so far remained elusive, despite some promising proof-of-principle results using X-ray wave-guiding ${ }^{[11,14]}$ and
X-ray reflectivity.[12] Microfluidic array phase profiling (MAPP) described here is complementary to most local probes like video microscopy and confocal microscopy, since it provides ensemble-averaged information that is spatially resolved across the confining average channel rather than data about an individual channel.

\section{Experimental}

Instead of investigating single fluid containers, our approach was to use an array of microfluidic channels etched into a silicon chip for small-angle X-ray scattering (SAXS) experiments. This approach has several advantages. By studying many identical containers at the same time, the ensemble average rather than a single entity is investigated, and the scattered X-ray signal is much stronger. The setup is insensitive to perturbations such as vibrations, since the dimensions of the fluid containers are not variable. Different dimensions of the confining channels are simply chosen by having a set of different arrays on the same sample, i.e. the same chip. The method is sensitive to electron density differences, i.e. no transparency or contrast of the components is needed in the visible light regime. 


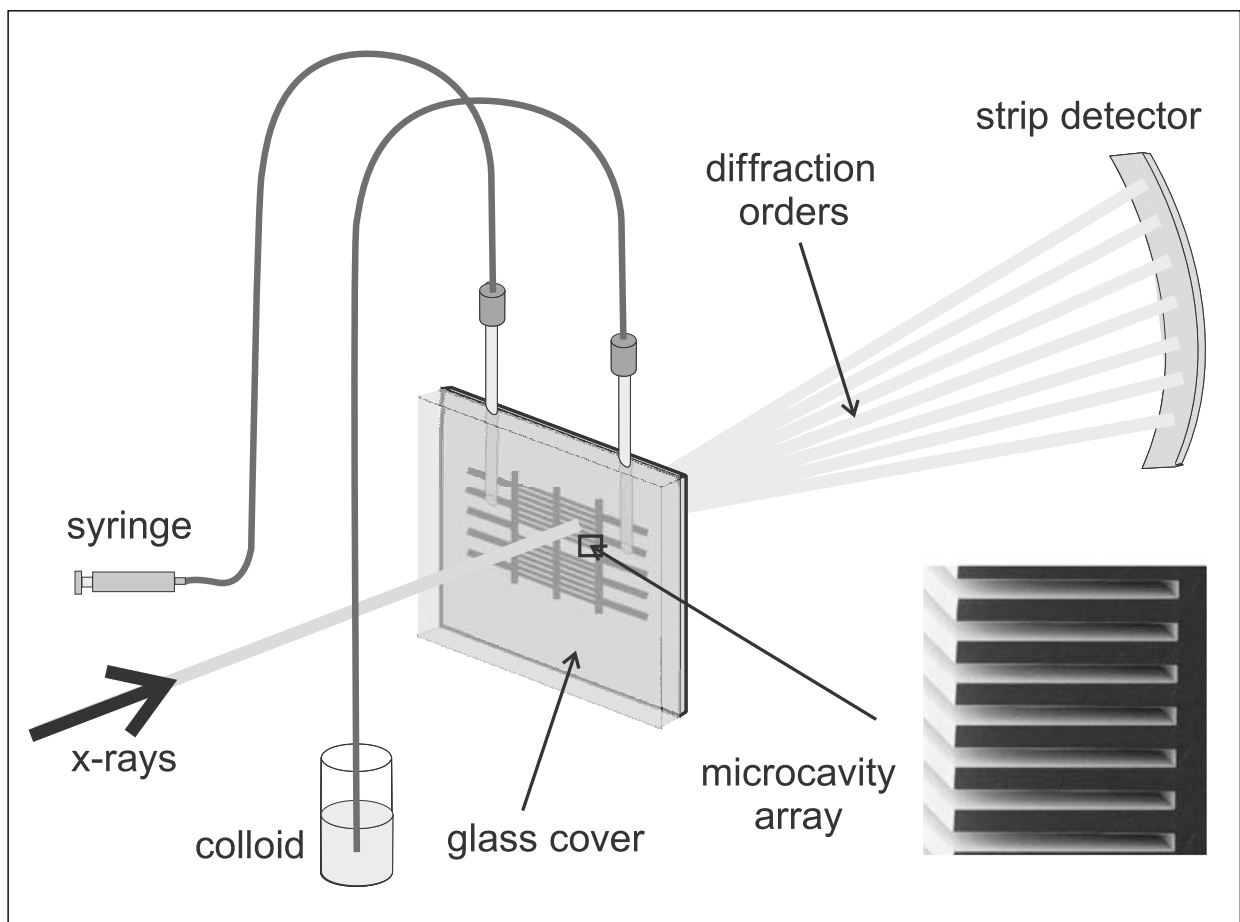

Fig. 1. Experimental setup. The X-ray beam impinges under normal incidence onto the sample chip on which several different microfluidic arrays were prepared. The sample chip is covered with a glass plate to avoid evaporation. The diffracted intensity is recorded with a high-resolution microstrip detector. The inset shows an SEM image of a microfluidic array.

Only the dimensions of the density fluctuations rather than the ordering constituents themselves have to be larger than the resolution. For example, ion concentrations can be measured.

We have succeeded in producing such arrays with a high degree of perfection. ${ }^{[15]}$ The data described in the present study were recorded for a chip with microfluidic arrays of $0.50,0.75,1.00$ and $1.25 \mu \mathrm{m}$ period and channel widths in the range of 280 to $615 \mathrm{~nm}$. The height of the structures is approximately $6 \mu \mathrm{m}$. Each array covers an area of $0.8 \mathrm{~mm} \times 0.8 \mathrm{~mm}$. A scanning electron microscopy (SEM) image of such a microfluidic array is shown in the inset of Fig. 1.

The microfluidic arrays were placed under normal incidence in the X-ray beam as schematically depicted in Fig. 1. The data were recorded at an X-ray energy of $11.804 \mathrm{keV}$, corresponding to a wavelength of $1.050 \AA$ at the Materials Science beam line of the Swiss Light Source, Paul Scherrer Institut.[16] A macroscopic beam of $0.5 \mathrm{~mm} \times 0.5 \mathrm{~mm}$ at the sample position and thereby most of the incident flux was used. The arrays act as diffraction gratings, and the intensities of the diffraction orders (Bragg peaks) for each grating were recorded with a high-resolution 1D micro-strip detector[17] having $10 \mu \mathrm{rad}$ resolution corresponding to a minimum resolvable momentum transfer of 6 $\times 10^{-4} \mathrm{~nm}^{-1}$. Combining three diffraction patterns obtained with different attenuation settings allowed us also to measure the low-order diffraction intensities, including the zeroth order, thereby eliminating the need for special treatment of missing lowfrequency data. ${ }^{[18,19]}$ The total exposure time for each microfluidic array was 135 seconds. This time already includes a triple repeat of each exposure used to filter out spurious counting events by taking the median value of the recorded intensities.

Because the illumination is only partially coherent, the X-ray data are sensitive to the average structure instead of individual channels and density profiles. In this sense, the current technique is more similar to crystallography than to lensless imaging using fully coherent illumination.

The charge-stabilized colloidal suspension consists of $10 \mathrm{vol} \%$ silica spheres of $109.2 \mathrm{~nm}$ average diameter in a refractiveindex matched solvent of $55 \%$ benzyl alcohol and $45 \%$ ethanol. The matching of the refractive index for the solution reduces differences in the electric susceptibility and thereby the van-der-Waals interactions. The polydispersity of the silica spheres is approximately $3 \%$. The concentration is well below the density for the formation of a crystalline layer at the wall or even capillary freezing. ${ }^{[20]}$ In a second measurement in the same microfluidic channels, lithium chloride $(\mathrm{LiCl})$ in 0.4 molar concentration was added. The additional $\mathrm{Li}^{+}$and $\mathrm{Cl}^{-}$ions present in the salt solution shield the electrostatic potential of the charged colloid particles. The silica colloid particles then effectively become similar to a hard-sphere system.

\section{Data Analysis}

For details of the data analysis we refer the interested reader to a previous publication. [21]

Fourier transformation of the recorded intensities of the grating orders cannot yield density profiles because of the well known phase problem, i.e. the loss of the $\mathrm{X}$ ray phase information in the recorded data. To retrieve the phase information, iterative algorithms have proven to be very helpful. The iterative phase-retrieval algorithm ${ }^{[21]}$ allows the determination of amplitude- and phase of the X-ray exit wave across the microfluidic channels in a model-independent way. Using the known refractive indices of a binary system one can directly calculate the concentration of, for example, colloidal particles in a solution.

Back propagation may be used to remove the container, i.e. the microfluidic array, when the problem is treated properly using dynamical theory. ${ }^{[22]}$ This approach has not been used for the data presented here.

For a model-dependent kinematic scattering approach to determine the concentration profile we refer to the literature. ${ }^{[23,24]}$

\section{Results and Discussion}

The results reported here are for colloidal solutions in the fluid phase. At higher concentrations, colloids form ordered solid phases. ${ }^{[25,26]}$ The phase profiles shown in the left panel of Fig. 2 are definitely far from being in the solid phase. Only weak modulations of the average density profile of the model fluid are visible. Dense crystalline ordering of the silica particles would lead to phase shifts much closer to the value of the surrounding silicon walls.

The most pronounced ordering effects are marked in Fig. 2 as circles, with the diameter of the colloidal particles of $109 \mathrm{~nm}$ drawn to scale. It is striking that only some of the channels exhibit ordering effects. For the solution with charged silica particles (left panel of Fig. 2) pronounced layer-like ordering is seen for the channels of $293 \mathrm{~nm}$ width whereas the $290 \mathrm{~nm}$ channels exhibit almost no ordering, and those with 304 nm probably show a mixture of several weaklyordered structures.

One may argue that for the charged colloidal particles, local charge differences are present and that the long-range forces lead to a variation in the ordering distance. The situation is better defined for colloids with salt solution. The ions in the salt solution shield the electrostatic potential, and the silica colloid particles effectively become a hard sphere system: the paradigm of a model fluid.

For the hard-sphere fluid, pronounced layering in two, three and four layers oc- 

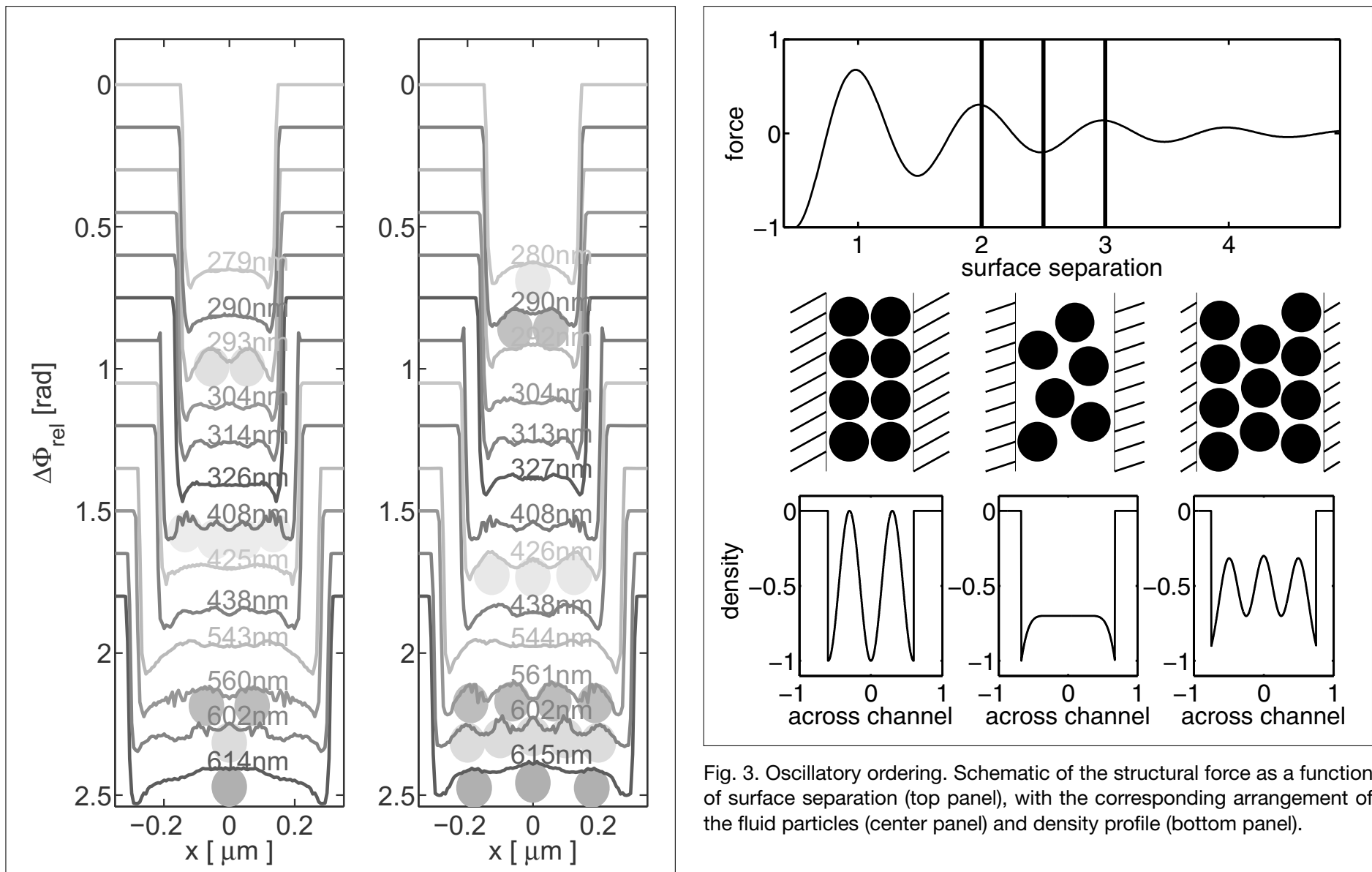

Fig. 3. Oscillatory ordering. Schematic of the structural force as a function of surface separation (top panel), with the corresponding arrangement of the fluid particles (center panel) and density profile (bottom panel).

Fig. 2. Phase profiles of charged silica colloids (left) and hard sphere like colloids (right) in the same microfluidic arrays. The profiles are offset vertically for clarity. The determined channel widths are shown for each profile. As we interpret oscillations as being caused by layering, i.e. increased concentration, we have highlighted these using spheres with the diameter of the colloid particles drawn to scale.

curs at multiples of approximately $142 \mathrm{~nm}$ in channel width, i.e. at 290, 426 and 561 $\mathrm{nm}$ (right panel of Fig. 2). This feature is a clear fingerprint of confinement-induced oscillatory ordering with a typical distance larger than the diameter of the colloidal particles of $109 \mathrm{~nm}$. Pronounced ordering is observed only if the given channel width fits to the layering distance of the colloidal particles inside. The data unequivocally show that the oscillatory structural forces are directly linked to a sequence of layering-disordering transitions, as illustrated in Fig. 3.

The concentration of the silica spheres, calculated from the phase profiles ${ }^{[21]}$ in Fig. 2 and spatially resolved across the confining channels, varies from $0 \%$ due to the excluded volume effect close to the walls to more than $25 \%$ in regions of high concentration (Fig. 4). For the charged colloidal particles (left panel), the depletion region close to the walls is larger than for the hard-sphere-like case (right panel). This increase is attributed to electrostatic repulsion. In addition, it is found that the average density across the channel width oscillates in phase with
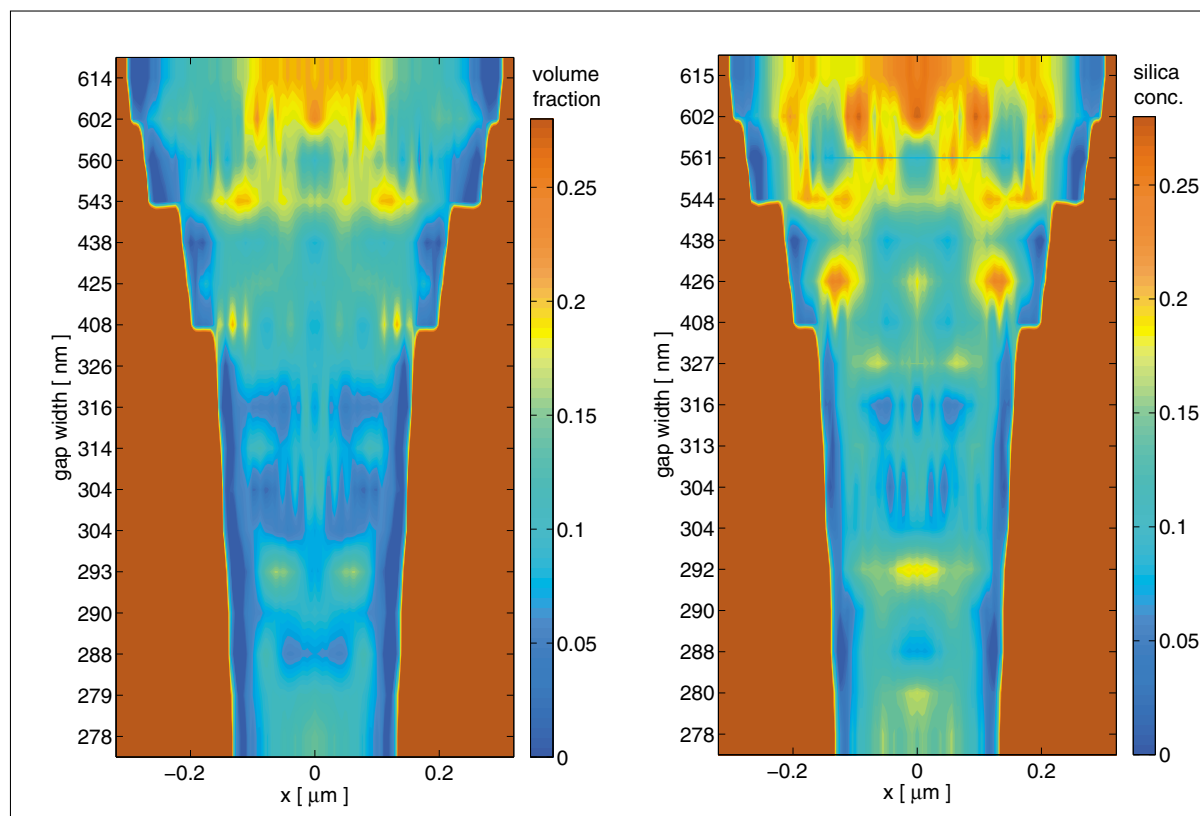

Fig. 4. Volume fraction of colloidal particles. The silica concentration inside the microfluidic channels is shown as a contour plot for several channel widths for charged silica colloids (left) and hardsphere-like colloids (right). The vol\% fraction directly at the confining walls as well as pronounced increases due to the confinement are clearly visible. The dark red outer region is the area of the confining silicon walls. 
the degree of order (not shown). This effect is not unexpected, since the layering effect allows for a denser packing of the particles. A similar oscillation in the average density has been observed in a recent optical study of a confined molecular fluid. ${ }^{[27]}$

At larger widths, the very regular layering breaks down. The density modulations induced by the walls no longer overlap sufficiently to induce well-ordered layering, even though the density within the channels is still significantly increased compared to other arrays having only small signs of ordering effects.

Intermediate density profiles, which we interpret as superpositions of different states of local ordering, are also observed for many gap sizes where an integer number of layers does not fit into the confining gaps, see for example the channels with 327 and $438 \mathrm{~nm}$ width in the right panel of Fig. 2 . This can be used to illustrate the complementarity of techniques: Local probes like video microscopy would find one or some of the realizations of the systems and this information can then be used to interpret the ensemble-averaged overall picture seen using the MAPP technique.

\section{Summary and Outlook}

We have identified oscillatory ordering of two, three and four layers in a silica hard-sphere model fluid confined in channels of sub-micron width. The colloidal solution nevertheless remains in the fluid state. A novel one-dimensional X-ray phase-retrieval approach applied to X-ray diffraction data from microfluidic arrays, coined MAPP, has enabled us to determine the oscillatory density profiles in a modelindependent way with a resolution in the 10 $\mathrm{nm}$ regime. This is the first time that the structural oscillation underlying the wellknown force oscillation has been observed directly. It would be very interesting to combine complementary techniques and study force and structure at the same time on the same sample.

The use of channel arrays for fluid confinement offers several advantages. Whereas a single channel typically contains less than a picoliter of fluid, an array has approximately one thousand times more fluid. This allows a correspondingly lower irradiation dose per volume for the same scattered signal. Lowering the dose per volume is important for systems that are prone to radiation damage (e.g. hydrocarbon-containing fluids). Secondly, the wetting of arrays of channels with different dimensions by the same fluid enables accurate studies of confinement effects, free of systematic errors. Thirdly, microfluidic arrays offer certain advantages for studies of dynamics using $\mathrm{X}$-ray photon correlation spectroscopy.[28]
Scaling down the channels in these arrays to the range of 10-50 nm will offer unique opportunities for studies of confinementinduced ordering of proteins in solution. These kind of systems can be studied since the MAPP technique is independent of the shape of the colloidal particles and for example rod-like particles or proteins are easily accessible.

\section{Acknowledgements}

This work was performed at the Swiss Light Source, Paul Scherrer Institut, Villigen, Switzerland. Hua (Tracy) Guo from the University of Amsterdam is gratefully acknowledged for assistance with the preparation of the colloidal solutions. We thank Dominik Meister and Michael Lange for technical support and Harun H. Solak and C. Theiler for fruitful discussions.

Received: May 1, 2008

[1] B. N. J. Persson, Comments Cond. Mat. Phys. 1995, 17, 281.

[2] B. Bhushan, J. N. Israelachvili, U. Landman, Nature 1995, 374, 607.

[3] W. J. Huisman, J. F. Peters, M. J. Zwanenburg, S. A. de Vries, T. E. Derry, D. Abernathy, J. F. van der Veen, Nature 1997, 390, 379.

[4] C.-J. Yu, A. G. Richter, A. Datta, M. K. Durbin, P. Dutta, Phys. Rev. Lett. 1999, 82, 2326.

[5] H. Reichert, O. Klein, H. Dosch, M. Denk, V. Honkimäki, T. Lippmann, G. Reiter, Nature 2000, 408, 839.

[6] J. F. van der Veen, H. Reichert, MRS Bulletin 2004, 29, 958.

[7] J. N. Israelachvili, 'Intermolecular and Surface Forces', 2nd ed., Academic Press, New York, 1991.

[8] T. Grünewald, C. A. Helm, Langmuir 1996, 12, 3885.

[9] P. Pieranski, L. Strzelecki, B. Pansu, Phys. Rev. Lett. 1983, 50, 900.

[10] D. H. van Winkle, C. A. Murray, J. Chem Phys. 1988, 89, 3885.

[11] M. J. Zwanenburg, J. H. H. Bongaerts, J. F. Peters, D. O. Riese, J. F. van der Veen, Phys. Rev. Lett. 2000, 85, 5154.

[12] O. H. Seeck, H. Kim, D. R. Lee, D. Shu, I. D. Kaendler, J. K. Basu, S. K. Sinha, Europhys. Lett. 2002, 60, 376.

[13] O. Bunk, A. Diaz, F. Pfeiffer, C. David, C. Padeste, H. Keymeulen, P. Willmott, B. Patterson, B. Schmitt, D. Satapathy, J. F. van der Veen, H. Guo, G. Wegdam, Phys. Rev. E 2007, 75, 021501.

[14] M. J. Zwanenburg, J. F. Peters, J. H. H. Bongaerts, S. A. de Vries, D. L. Abernathy, J. F. van der Veen, Phys. Rev. Lett. 1999 , 82, 1696.

[15] A. Diaz, C. David, H. Guo, H. Keymeulen, F. Pfeiffer, G. Wegdam, T. Weitkamp, J. F. van der Veen, Physica B 2005, 357, 199.

[16] B. D. Patterson, R. Abela, H. Auderset, Q. Chen, F. Fauth, F. Gozzo, G. Ingold, H Kühne, M. Lange, D. Maden, D. Meister, P. Pattison, Th. Schmidt, B. Schmitt, C. Schulze-Briese, M. Shi, M. Stampanoni, P. R. Willmott, Nucl. Instr. Meth. in Phys. Res. A 2005, 540, 42
[17] B. Schmitt, C. Brönnimann, E. F. Eikenberry, G. Hülsen, H. Toyokawa, R. Horisberger, F. Gozzo, B. Patterson, C. SchulzeBriese, T. Tomizaki, Nucl. Instr. Meth. in Phys. Res. A 2004, 518, 436.

[18] D. Shapiro, P. Thibault, T. Beetz, V. Elser, M. Howells, C. Jacobsen, J. Kirz, E. Lima, H. Miao, A. M. Neiman, D. Sayre, Proc. Natl. Acad. Sci. USA 2005, 102, 15343.

[19] P. Thibault, V. Elser, C. Jacobsen, D. Shapiro, D. Sayre, Acta Cryst. A 2006, 62, 248.

[20] M. Dijkstra, Phys. Rev. Lett. 2004, 93, 108303.

[21] O. Bunk, A. Diaz, F. Pfeiffer, C. David, D. K. Satapathy, J. F. van der Veen, Acta Cryst. A 2007, 63, 306.

[22] K. Nygård, D. K. Satapathy, O. Bunk, F. Pfeiffer, C. David, J. F. van der Veen, Appl. Phys. Lett. 2008, 92, 214105.

[23] A. Diaz, J. F. van der Veen, Thin Solid Films 2007, 515, 5645.

[24] A. Diaz, 'X-Ray Scattering Studies of Ordering Phenomena in Colloids Confined in Microcavity Arrays', Thesis, no. 16851, ETH Zurich, 2006.

[25] P. N. Pusey, W. van Megen, Nature 1986, 320, 340.

[26] E. B. Sirota, H. D. Ou-Yang, S. K. Sinha, O. M. Chaikin, J. D. Axe, Y. Fujii, Phys. Rev. Lett. 1989, 62, 1524.

[27] M. Heuberger, M. Zäch, Langmuir 2003, 19, 1943.

[28] D. O. Riese, G. H. Wegdam, W. L. Vos, R. Sprik, D. Fenistein, J. H. H. Bongaerts, G. Grübel, Phys. Rev. Lett. 2000, 85, 5460. 